\title{
An empowerment information intervention improved participation in treatment decision making in men with recently diagnosed prostate cancer
}

Davison BJ, Degner LF Empowerment of men newly diagnosed with prostate cancer. Cancer Nurs 1997 Jun;20:187-96.

\section{Objective}

To determine whether assisting men with recently diagnosed prostate cancer to obtain relevant information increases their participation in treatment decision making, and decreases anxiety and depression.

\section{Design}

Randomised controlled trial with 6 week follow up.

\section{Setting}

A community urology clinic in Winnipeg, Manitoba, Canada.

\section{Patients}

60 consecutive men (mean age 68 y) with a recent diagnosis of prostate cancer, who had not been told their diagnosis; had not had their initial treatment consultation; were able to speak, read, and write English; and showed no evidence of mental confusion. $87 \%$ were married, $72 \%$ were retired, and $58 \%$ had less than a grade 12 education.

\section{Intervention}

Men allocated to the intervention $(n=30)$ received 5 brochures containing information on, for example, the disease process, treatment options, and diagnostic tests, and were instructed on how to find information in the brochures. A list of potential questions for discussion with the physician was reviewed, new questions raised by the patient were added, and a copy of the final list was given to the patient. Patients received a blank audiotape to tape the consultation, and were responsible for asking the physician to tape the meeting. They were encouraged to participate in decisions about treatment options, and to bring their significant others to the meeting. Men allocated to the control group $(n=30)$ received the same 5 brochures, were shown what they contained, and were told it might be helpful if they reviewed the brochures before or after the initial consultation.

\begin{abstract}
Main outcome measures
Patients' preferred roles in treatment decision making were assessed before the intervention, using a card sort technique. Men were presented with 5 cards that each presented different roles that they could assume with respect to their treatment decision making, and were asked to select the 1 card that best represented their preferences. Based on their selection, patients were classified as active, passive, or collaborative. 6 weeks after the intervention, actual roles assumed during the consultation were assessed using the same card sort task. Anxiety, measured using the Spielberger State-Trait Anxiety Inventory, and depression, measured using the Centre for Epidemiologic Studies Depression Scale, were assessed before the intervention and at 6 weeks after the consultation.
\end{abstract}

\section{Main results}

More men in the intervention group assumed an active role during the consultation than men in the control group $(57 \% v$ $17 \%, \mathrm{p}<0.001)$ and there were no differences between groups for men who assumed collaborative or passive roles. The groups did not differ for state or trait anxiety levels, or mean depression scores at 6 weeks.

\section{Conclusion}

More men with recently diagnosed prostate cancer who received an intervention to encourage and support participation in treatment decisions assumed an active role during an initial physician consultation, but were not less anxious or depressed.

Sources of funding:National Cancer Institute of Canada with funds provided by the Canadian Cancer Society.

For article reprint: B Joyce Davison, St. Boniface General Hospital Research Centre, 351 Tache Avenue, Winnipeg, Manitoba R2H 2A6, Canada.Fax +1 2042314006.

\section{Commentary}

Research shows that oncology patients are often dissatisfied with the type and amount of information that they receive, and have difficulty participating meaningfully in decision making. In an age of rapid scientific strides, changing treatment protocols, and widely publicised debates about what constitutes optimal cancer treatment, this study by Davison and Degner is timely and important.

The study was based on a sound theoretical structure, used random assignment to intervention or control group, and adhered to standardised protocols. As such, the study should serve as a prototype for future research in this area. A small sample size, however, with only 30 men in each group, prevents the drawing of any firm conclusions. The authors state that the groups were demographically similar at baseline. Although not statistically sig- nificant, at a glance, the intervention group had a lower mean age, included more younger men, a wider age distribution, and fewer retirees than the control group. Because of an inadequate sample size, random assignment was ineffective in its task of producing similar groups. Interestingly though, these differences could not be detected statistically, again because of small numbers. It is possible that the significant difference in state anxiety at baseline between the two groups was caused by these undetected differences. The authors cite a study of newly diagnosed patients with cancer which found the lowest levels of psychological distress among the oldest individuals. ${ }^{2}$

This study clearly makes a case for empowering men to be involved in decision making, although the impact of the intervention on anxiety and depres- sion (prominent symptoms at cancer diagnosis) is unclear. Again, a larger sample may permit conclusions to be drawn. It may be, however, that anxiety and depression are outcomes that do not respond to the intervention in question or that a "psychological" component must be added to the treatment arm. Finally, it cannot be assumed that participation in treatment decisions is similar across cultures, justifying replication of the study among diverse groups.

Jeannie V Pasacreta, RN, CS, PhD Associate Professor Yale University School of Nursing New Haven, Connecticut, USA

1 Degner LF, Sloan JA. Decision making during serious illness: what role do patients really want to play? J Clin Epidemiol 1992;45:941-50.

2 Edlund, B, Sneed NV. Emotional responses to the diagnosis of cancer: age-related comparisons. Oncol Nurs Forum 1989;16:691-7. 